OPEN ACCESS

Edited by:

Lifeng Zhu,

Nanjing Normal University, China

Reviewed by:

Mohsin Khurshid,

Government College University,

Faisalabad, Pakistan

Muhammad Tahir UI Qamar,

Guangxi University, China

${ }^{*}$ Correspondence:

Bin Li

libin0571@zju.edu.cn

tThese authors have contributed equally to this work

Specialty section:

This article was submitted to

Microbial Symbioses,

a section of the journal

Frontiers in Microbiology

Received: 26 May 2021

Accepted: 29 June 2021

Published: 05 August 2021

Citation:

Zhou G, Yu R, Ahmed T, Jiang H,

Zhang M, LV L, Alhumaydhi FA,

Allemailem KS and Li B (2021) Biosynthesis and Characterization of Zinc Oxide Nanoparticles and Their Impact on the Composition of Gut Microbiota in Healthy and Attention-

Deficit Hyperactivity Disorder Children.

Front. Microbiol. 12:700707. doi: 10.3389/fmicb.2021.700707

\section{Biosynthesis and Characterization of Zinc Oxide Nanoparticles and Their Impact on the Composition of Gut Microbiota in Healthy and Attention- Deficit Hyperactivity Disorder Children}

\author{
Guoling Zhou ${ }^{1 \dagger}$, Rongrong Y ${ }^{2 t}$, Temoor Ahmed ${ }^{3}$, Hubiao Jiang ${ }^{3}$, Muchen Zhang ${ }^{3}$, \\ Luqiong Lv ${ }^{3}$, Fahad A. Alhumaydhi ${ }^{4}$, Khaled S. Allemailem ${ }^{4}$ and Bin $\mathrm{Li}^{3 *}$
}

\begin{abstract}
${ }^{1}$ Hangzhou Seventh People's Hospital (HSPH), Hangzhou, China, ${ }^{2}$ School of Education Science and Technology, Zhejiang University of Technology, Hangzhou, China, ${ }^{3}$ Institute of Biotechnology, Zhejiang University, Hangzhou, China, ${ }^{4}$ Department of Medical Laboratories, College of Applied Medical Sciences, Qassim University, Buraydah, Saudi Arabia
\end{abstract}

Attention-deficit hyperactivity disorder (ADHD) seriously affects children's health, and the gut microbiome has been widely hypothesized to play a role in the regulation of ADHD behavior. The present study aims to the biosynthesize of zinc oxide nanoparticles (ZnONPs) by using Acinetobacter johnsonii strain RTN1, followed by their characterization through state-of-the-art material characterization techniques, viz., UV-vis spectroscopy, Fourier transform infrared spectroscopy, and transmission and scanning electron microscopic analyses with energy dispersive spectrometry. Moreover, we investigated and compared the population composition of gut microbiota and their susceptibility to biogenic ZnONPs between healthy and ADHD children based on the traditional plate method and 16S rRNA amplicon sequence analysis. The antibacterial effect of ZnONPs against gut bacteria was also determined by measurement of live cell number, living/dead bacterial staining test, and flow cytometry observation. The present study revealed that the number of live gut bacteria in healthy children was more than 10-fold higher than that in ADHD children; however, the community structure of gut bacteria has changed, while greater diversity was found in gut bacteria from $A D H D$ children. In addition, we found that the number of live gut bacteria in healthy and ADHD children was reduced by ZnONPs, which shows an increased and reduced effect in composition of gut bacteria from healthy and ADHD children, respectively. It was also noted that the main mechanism of ZnONPs may be to inhibit the growth of gut bacteria rather than to kill them, while the nanoparticle-resistant strains in healthy children is also different from that in ADHD children. Some representative bacteria, in particular nanoparticle-resistant bacteria, were successfully isolated and identified. Overall, this study revealed the potential correlation of ADHD with gut bacteria and provided a new possibility to prevent $A D H D$ by the combination of nanoparticle and its resistant bacteria.

Keywords: attention-deficit hyperactivity disorder, amplicon sequencing, bacterial community, gut bacteria, zinc oxide nanoparticles 


\section{INTRODUCTION}

Attention-deficit hyperactivity disorder (ADHD) is one of the most prominent developmental and neuropsychiatric disorders characterized by problems with inattention, impulsivity, and hyperactivity, which have been increasingly diagnosed in the last decade and affects approximately $8-12 \%$ of school-aged children worldwide (Luo et al., 2019). Children with ADHD are extremely active and unable to concentrate on anything for very long, with the result that they find it difficult to learn and often behave in inappropriate ways (Wang et al., 2020). Although pharmacological treatments can reduce symptoms, they are often unsatisfactory due to side effects, fail to prevent or alter long-term course, and are discontinued due to patient and family preferences (Hiergeist et al., 2020). Several studies have reported that the incidence of ADHD may have a certain degree of heritability, and the abnormally expressed genes in children with ADHD genes are associated with norepinephrine and serotonin [5-hydroxytryptamine (5-HT)] as well as dopamine synthesis and transmission (Karmakar et al., 2017), Furthermore, the mutual effects of environmental factors and genetic polymorphism are also attracting much attention in ADHD studies (Hayashi and Iwanami, 2018). Although various theories have been proposed, the pathogenic mechanisms underlying ADHD have not been fully clarified.

Interestingly, there is also growing evidence supporting the role of the human gut microbiome in the development of ADHD (Sampson and Mazmanian, 2015; Boonchooduang et al., 2020). It has been well known that the gut microbiota is the largest ecosystem in the human body, which is believed to play a pivotal role in human health and disease such as psychiatric disorders in multiple ways (Martins-Silva et al., 2021). In practicality, the brain-gut axis theory proposes that the gut microbiota can influence brain function and behaviors through involvement in physiological homoeostasis, immunological development, glutathione metabolism, amino acid metabolism, etc (De Vadder et al., 2014). Therefore, it is necessary for us to investigate the change in the composition of gut microbiome from ADHD children, which will provide a new insight to elucidate the mystery of this complex disease.

In recent years, green synthesized nanomaterials have gained much attention because of their potential use as therapeutic agents (Kummara et al., 2016; Alphandéry, 2020). Although many physical and chemical approaches have been used to produce nanomaterials, the microbe-mediated synthesis of zinc oxide nanoparticles (ZnONPs) is more favorable than traditional methods because of their unique physicochemical properties such as crystalline structure, surface area, size, and shape, and more stable nature (Ahmed et al., 2021c). Recently, biologically synthesized nanoparticles (NPs) have been well documented and served as the best alternative to previously available expensive and environment-damaging physicochemical technologies (Ahmed et al., 2020, 2021a). Indeed, our previous studies have indicated that green synthesized metallic NPs have potential bacteriodical effect against human pathogenic Gram-positive bacteria Staphylococcus aureus and gram-negative bacteria
Escherichia coli (Ahmed et al., 2020). Although the evaluation of microbiota/microbiome-mediated effects of nanomaterials is in its early infancy, few experimental studies have been carried out on the interaction between nanomaterials and gut microbiome/microbiota, which revealed a double-face effect in the relation between nanomaterials and human health with possible adverse effects from one side and with possible beneficial application on the other (Pietroiusti et al., 2016). These differential results may be mainly attributed to the difference in the type, time, and dose of the used NPs. In previous studies, biosynthesized $\mathrm{ZnONPs}$ are described as being biocompatible and as not inducing cytotoxicity toward healthy cells (Agarwal et al., 2018; Saravanan et al., 2018; Suresh et al., 2018).

The aim of this study was to investigate the population number and composition of gut microbiota from healthy and ADHD children and their susceptibility to biogenic ZnONPs based on the analysis of 16S rRNA amplicon sequencing in a combination of the plate counting method, living/dead bacteria staining test, flow cytometry observation, and the16S rDNA sequence analysis. The result of this study will provide a new insight for us to prevent ADHD by the combined use of NP its resistant bacteria.

\section{MATERIALS AND METHODS}

\section{Biosynthesis and Characterization of ZnONPs}

Fresh culture of Acinetobacter johnsonii strain RTN1 (accession no. MT173803) was collected from the culture collection of the Institute of Biotechnology, Zhejiang University, China. The ZnONPs were synthesized by using bacterial strain RTN1, according to Selvarajan and Mohanasrinivasan (2013). In brief, the bacterial strain RTN1 was grown overnight in nutrient broth (NB) media at $28 \pm 2{ }^{\circ} \mathrm{C}$ in a shaking incubator, and supernatant of bacterial culture was collected after centrifugation at $5,000 \mathrm{~g}$ for $10 \mathrm{~min}$. For the biosynthesis of ZnONPs, a ratio of $1: 1$ of $0.1 \mathrm{M} \mathrm{ZnSO}_{4} \cdot 7 \mathrm{H}_{2} \mathrm{O}$ and the culture supernatant of strain RTN1 were mixed in a 250-ml Erlenmeyer flask, and the reaction mixture was subjected to heating on a water bath up to $80^{\circ} \mathrm{C}$ for $15 \mathrm{~min}$. The visible white precipitates that appeared at the bottom of the flask confirmed the synthesis of ZnONPs. Afterward, ZnONPs were collected by centrifugation at $10,000 \mathrm{~g}$ for $10 \mathrm{~min}$ and washed three times with distilled water.

The synthesis of ZnONPs in a reaction mixture was observed using a UV-vis spectrophotometer (Shimadzu Corporation, Kyoto, Japan) at 300- to $700-\mathrm{nm}$ wavelength range (Ahmed et al., 2021c). The Fourier transform infrared spectroscopy (FTIR) analysis was carried out to identify different functional groups present in the ZnONPs by using a FTIR spectrometer (Vector 22; Bruker, Germany) in the spectral region range of $4,000-500 \mathrm{~cm}^{-1}$. Moreover, the size, shape, and element composition of biogenic ZnONPs were observed using scanning electron microscopy (SEM; SU8010, Hitachi, Japan) and transmission electron microscopy (TEM; JEM-1230, JEOL, Akishima, Japan) analyses. Moreover, the elemental composition of $\mathrm{ZnONPs}$ was confirmed through energy-dispersive 
spectroscopy (EDS; Oxford, United Kingdom) at $20 \mathrm{keV}$, according to Ahmed et al. (2021b).

\section{Collection of Fecal Samples}

In this study, we recruited a total of 44 children with ADHD (mean age: 6.9 years) who were treated in the Pediatric Outpatient Department of Hangzhou Seventh People's Hospital from January 2019 to July 2020. In addition, a total of 38 healthy children were recruited from primary schools in Hangzhou in this study as the control (mean age: 8.6 years) for analysis of fecal microbiota. All children were between 6 and 12 years old, while the identities of the ADHD children were determined in Hangzhou Seventh People's Hospital based on the current criteria of the DSM-5 ("Diagnosis and Statistical Mannual of Mental Disorders, Fifth Edition"). After collecting the feces from each recruited children in the corresponding centrifuge tube and then putting each sample in an ice bag, all fecal samples were stored at $-20^{\circ} \mathrm{C}$ for further use.

\section{Preparation of the Mixed Fecal Samples}

The mixed fecal samples of ADHD children were prepared by taking about $1.0 \mathrm{~g}$ of fresh fecal samples from each ADHD children and then dissolving them into $10 \mathrm{ml}$ of sterile distilled water. Following the thorough shaking and mixing and centrifugation at $10,000 \mathrm{~g}$ for $3 \mathrm{~min}$ at room temperature, the pellets were collected and stored at $4^{\circ} \mathrm{C}$ for further use. A similar procedure was applied for the preparation of the mixed fecal samples of healthy children.

\section{Susceptibility of Gut Microbiota to ZnONPs}

The suspension of gut microbiota was prepared by dissolving the mixed fecal samples from ADHD and healthy children in distilled water. Susceptibility of gut microbiota to ZnONPs was determined by adding the stock solution $(1.0 \mathrm{mg} / \mathrm{ml})$ of ZnONPs into the suspension of the mixed fecal samples to obtain a final concentration of $20 \mu \mathrm{g} / \mathrm{ml}$.

\section{Counting Surviving Bacterial Cells}

Suspensions of gut bacteria were prepared by dissolving the mixed fecal samples from ADHD and healthy children in distilled water and then filtering with four layers of gauze to remove impurities. The surviving bacterial cells were counted as described by Li et al. (2008). In brief, gut bacterial suspensions were 10 -fold serially diluted, and $10-\mu$ samples were inoculated on nutrient agar medium in sextuplicate for each dilution and were incubated for $48 \mathrm{~h}$ at $37^{\circ} \mathrm{C}$. After incubation, the surviving cells on the agar were counted based on the colony-forming units, and then the mean value of the cells at the lowest dilution was calculated. This experiment was repeated twice with six replicates of each treatment.

\section{Live/Dead Cell Staining}

To observe the damage and intact membranes in cells of gut bacteria exposed to $20 \mu \mathrm{g} / \mathrm{ml} \mathrm{ZnONPs,} \mathrm{we} \mathrm{performed} \mathrm{an}$ analysis of live/dead cell staining. In brief, the mixed fecal samples from ADHD and healthy children were dissolved in distilled water and then filtered with four layers of gauze to remove impurities. Live/dead staining of gut bacteria was carried out as described by Yu et al. (2020) according to the protocol of BacLight bacterial viability kit (Invitrogen) following the induction of isopropyl-beta-D-thiogalactopyranoside (IPTG) with the final concentration of $1 \mathrm{mM}$. There are two nucleic acid stains in the kit named (i) red fluorescent (propidium iodide stain) for dead bacteria and (ii) green fluorescent (SYTO 9 stain) for live bacteria. Fluorescence in the sample was detected using the Olympus inverted confocal microscope.

\section{Flow Cytometry Observation}

Flow cytometry was detected as described by Ogunyemi et al. (2019b) with minor revision. In brief, after IPTG was induced for 12 and $24 \mathrm{~h}$, the harvested bacteria were centrifuged at $5,000 \mathrm{~g}$ for $10 \mathrm{~min}$, then washed three times using $\mathrm{ddH}_{2} \mathrm{O}$, and, finally, the PI solution was diluted in bacterial suspension to $50 \mathrm{mg} / \mathrm{L}$, and the bacteria were stained for $20 \mathrm{~min}$ in the dark and then washed. The staining cells can be monitored using flow cytometry (Beckman Coulter, Gallios, Germany).

\section{S rRNA Amplicon Sequencing Analysis}

\section{Extraction of Genomic DNAs}

Total DNA was extracted from stool samples using the OMEGA Soil DNA Kit (D5625-01; OMEGA Bio-Tek, Norcross, GA, United States) according to the manufacturer's recommendations for human stool samples. The quantity and quality of the extracted DNA were detected by the NanoDrop ND-1000 spectrophotometer (Thermo Fisher Scientific, Waltham, MA, United States) and agarose gel electrophoresis, respectively. Extracted DNA was stored at $-20^{\circ} \mathrm{C}$ until PCR amplifcation and Illumina MiSeq DNA sequencing.

\section{S rRNA Amplicon Sequencing}

The DNA samples were subjected to the first-run PCR, where the specified forward primer 338F (5'-ACTCCTACGGGAG CAGCA-3') and reverse primer 806R (5'-GGACTACHVGGGTW TCTAAT-3') were designed to amplify the V3-V4 genomics region of bacterial $16 \mathrm{~S}$ rRNA genes. The PCR system contain $5 \mu \mathrm{l}$ of buffer solution ( $5 \times), 0.25 \mu \mathrm{l}$ of fast pfu DNA polymerase $(5 \mathrm{U} / \mu \mathrm{l}), 2 \mu \mathrm{l}$ of dNTPs $(2.5 \mathrm{mM}), 1 \mu \mathrm{l}$ of forward and reverse primers $(10 \mu \mathrm{M}), 1 \mu \mathrm{l}$ of DNA template solution, and $14.75 \mu \mathrm{l}$ of $\mathrm{ddH}_{2} \mathrm{O}$. The thermal cycle consists of an initial denaturation of $5 \mathrm{~min}$ at $98^{\circ} \mathrm{C}$, followed by 25 cycles of denaturation at $98^{\circ} \mathrm{C}$ for $30 \mathrm{~s}$, annealing at $53^{\circ} \mathrm{C}$ for $30 \mathrm{~s}$, expansion at $72^{\circ} \mathrm{C}$ for $45 \mathrm{~s}$, and, finally, an extension of $5 \mathrm{~min}$ at $72^{\circ} \mathrm{C}$. The PCR products were purified by using $\mathrm{V}$ azyme VAHTSTM DNA Clean Beads (V azyme, Nanjing, China) and quantified by Quant-iT PicoGreen dsDNA detection kits (Invitrogen, Carlsbad, CA, United States). Following the confirmation with gel electrophoresis and polymerization in equal quantities, the PCR-amplified products were then subjected to library preparation for $16 \mathrm{~S}$ rRNA sequencing using the $2 \times 250$-bp paired-end protocol. We prepared a DNA library according 
to the 16S rRNA Sequencing Library Preparation instructions (Illumina, CA, United States). The prepared amplicons were sequenced on the MiSeq platform (Illumina, CA, United States) with the MiSeq Reagent Kit v3 (600 cycles) from Shanghai Personal Biotechnology Co. Ltd. (Shanghai, China).

\section{Bioinformatics Analysis}

Sequence data analysis was carried out as described by Stevens et al. (2019) with minor modification using QIIME2 (version 2020.06) and R package (v3.6.0). In brief, 16S rRNA gene amplicon sequences were demultiplexed and preprocessed. Barcode and primer removal, quality control, amplicon sequence data correction, phiX filtering, and dereplication were verified using the DADA2 sofware package43 (Callahan et al., 2016). A phylogenetic tree was constructed with fasttree2. The structural changes of the microbial community in different samples were investigated by calculating the alpha diversity index, which was determined by using Shannon, Simpson, Pielou's evenness, beta diversity index including weighted and unweighted UniFrac, Jaccard distance, and Bray-Curtis. Comparing the richness and evenness of amplicon sequence variant (ASV) among samples, each sample is limited to the same number of reads $(33,680$ sequences, the smallest number of sequences). Principal components analysis (PCA) was performed on the phylum, genus, and species levels of each sample.

\section{Statistical Analysis}

The software STATGRAPHICS Plus, version 4.0 (Copyright Manugistics Inc., Rockville, MD, United States) was used to perform the statistical analyses. Levels of significance $(p<0.05)$ of main treatments and their interactions were calculated by the analysis of variance (ANOVA) after testing for normality and variance homogeneity.

\section{RESULTS AND DISCUSSION}

\section{Biosynthesis and Characterization of ZnONPs}

In the present study, biogenic ZnONPs were extracellularly synthesized by using a bacterial strain RTN1, and the maximum precipitate clustered at the bottom of the flask was determined at $0.1 \mathrm{M} \mathrm{ZnSO}_{4} \cdot 7 \mathrm{H}_{2} \mathrm{O}$ concentration. Similarly, Ahmed et al. (2021c) observed the extracellular biosynthesis of ZnONPs from Bacillus cereus as a capping and reducing agent. Several studies reported that the microbe-mediated synthesis of NPs are eco-friendly, less toxic, and more stable as compared with chemical synthesis (Gericke and Pinches, 2006; Ali et al., 2020). The production of ZnONPs was further confirmed by the UV-vis absorption peak measured at $361 \mathrm{~nm}$ (Figure 1A), which is in agreement with the study of Malaikozhundan et al. (2017), who synthesized the Bacillus thuringiensis-coated ZnONPs and observed the UV-vis spectral peak at $373 \mathrm{~nm}$, which is consistent with our study.

The FTIR analysis was used to confirm the presence of different functional groups involved in the long-term stabilization of biogenic ZnONPs. The FTIR spectra of ZnONPs showed the absorption peaks at 3,423,1,637, 1,406, and $1,058 \mathrm{~cm}^{-1}$ and a weak peak at $560 \mathrm{~cm}^{-1}$ (Figure 1B). The strong peaks at 3,423 and $1,637 \mathrm{~cm}^{-1}$ were revealed in the presence of the hydroxyl group $(-\mathrm{OH})$ of alcohol and $\mathrm{C}=\mathrm{C}$ stretching of alkene, respectively. The peaks at 1,406 and $1,058 \mathrm{~cm}^{-1}$ were attributed to the stretching of $\mathrm{O}-\mathrm{H}$ bending and $\mathrm{C}-\mathrm{O}$ stretching of the alcohol group, respectively. Our FTIR results are consistent with the findings of Selvarajan and Mohanasrinivasan (2013), who biologically produced the ZnONPs from the Lactobacillus plantarum VITES07. Overall, it can be concluded that these functional groups as capping agents might act as a barrier to prevent agglomeration among ZnONPs and maintain their long-term stability. Moreover, the SEM and TEM analyses revealed that biogenic ZnONPs have spherical shapes with particle size ranging from 22 to $46 \mathrm{~nm}$ (Figure 2). The results of the EDS spectral analysis confirmed the presence of zinc (56.73\%), oxygen $(38.25 \%)$,

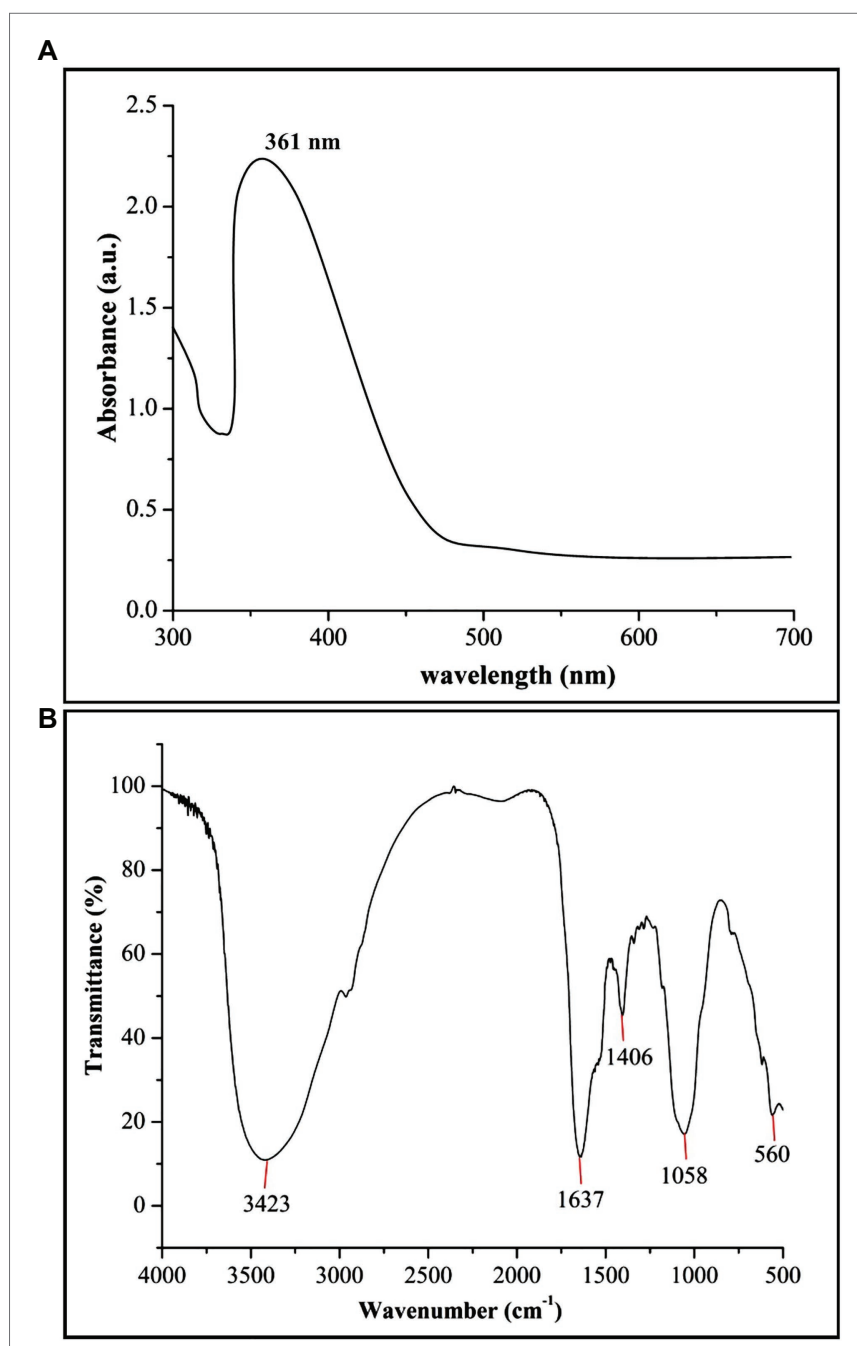

FIGURE 1 | Characterization of biogenic zinc oxide nanoparticles (ZnONPs) using (A) UV-vis spectroscopy and (B) Fourier transform infrared spectroscopy (FTIR) analysis. 
aluminum (0.82\%), sulfur (2.90\%), and silicon (1.31\%) in biogenic ZnONPs (Figure 2C), which is consistent with the previous studies (Ogunyemi et al., 2019a, 2020).

\section{Changes of Gut Bacteria by Counting the Surviving Cells}

The latest research in childhood ADHD has slowly shifted from just concerned about genetics to more and more focus on the gut bacteria, which play an important role (BundgaardNielsen et al., 2020; Checa-Ros et al., 2021). Indeed, changes in gut microbiota profiles in children with ADHD have been reported in several studies (Hiergeist et al., 2020). In order to assess the possible role of the gut microbiota in disease development and to open new avenues for ADHD treatment, we conducted a case-control study to investigate and compare the number and composition of gut bacteria from the fecal samples of healthy and ADHD children based on the traditional plate method and a $16 \mathrm{~S}$ rRNA amplicon sequencing approach. In addition, we investigated the antibacterial effect of $\mathrm{ZnONPs}$ against gut bacteria by measurement of live cell number, living/ dead bacterial staining test, and flow cytometry observation, while the change in composition of gut microbiome by $\mathrm{ZnONPs}$ was determined by sequencing of $16 \mathrm{~S}$ rRNA gene from the obtained resistant strains and $16 \mathrm{~S}$ rRNA amplicon sequencing of fecal samples from healthy and ADHD children. Overall, the results revealed the importance of gut bacteria in ADHD and gave a new strategy to prevent ADHD by the combination of NP and its resistant bacteria.

The results from this study indicated that in the absence of $\mathrm{ZnONP}$, the number of the live bacteria is $6.95 \times 10^{8} \mathrm{CFU} / \mathrm{g}$ fresh feces from healthy children, while there was a $91.75 \%$ reduction in the number of the live bacteria in fresh feces from children with ADHD compared to the healthy children. Furthermore, the addition of $\mathrm{ZnONP}$ with a final concentration of $20 \mu \mathrm{g} / \mathrm{ml}$ caused a $96.69 \%$ reduction in the number of live bacteria in fresh feces from healthy children. In comparison, the addition of $\mathrm{ZnONP}$ with a final concentration of $20 \mu \mathrm{g} /$ $\mathrm{ml}$ caused a $96.73 \%$ reduction in the number of live bacteria in fresh feces from children with ADHD compared to the healthy children (Figure 3). The result of this study indicated that ADHD caused a significant reduction in the number of live gut bacteria, which is consistent with the result of several previous studies (Stevens et al., 2019; Szopinska-Tokov et al., 2020; Wan et al., 2020). Furthermore, our results also indicated that
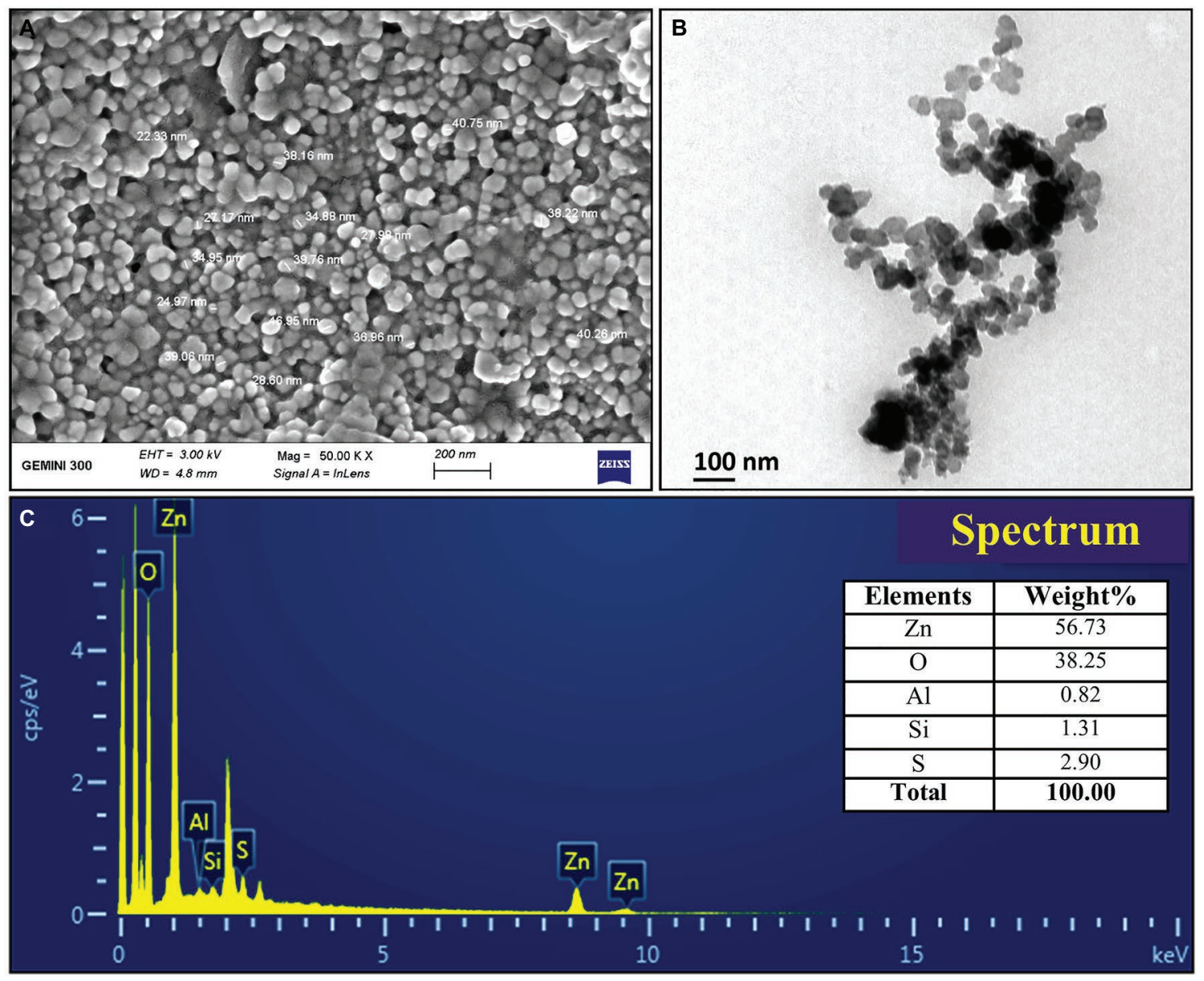

FIGURE 2 | Characterization of biogenic ZnONPs using (A) scanning electron microscopy (SEM), (B) transmission electron microscopy (TEM), and (C) EDX spectrum. 
the number of live gut bacteria was significantly reduced by NPs regardless of healthy or ADHD children. In agreement with the data of this study, Abid et al. (2020) reported that the biosynthesized iron oxide NPs had strong antibacterial

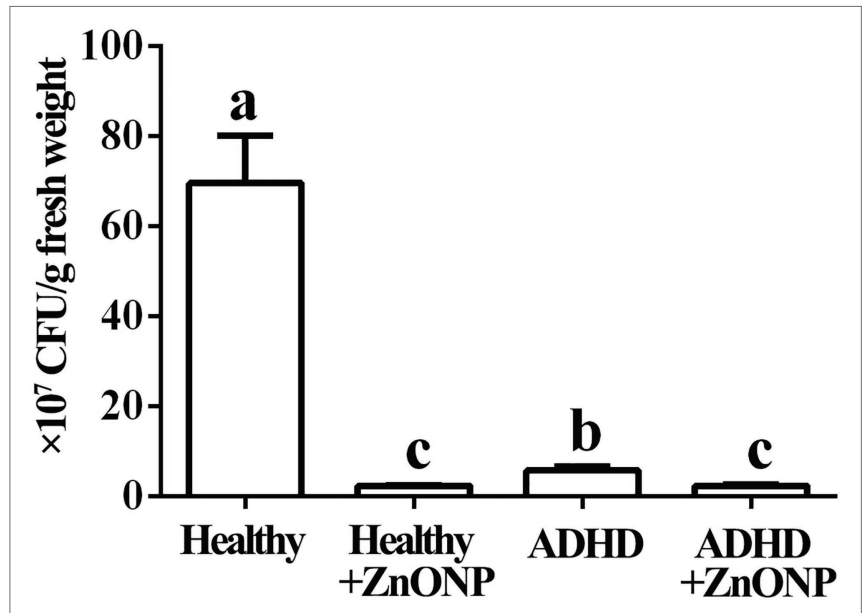

FIGURE 3 | Numbers of gut bacteria in fecal samples from the healthy and attention-deficit hyperactivity disorder (ADHD) children by the plate counting method. Vertical bars represent standard errors of the means $(n=6)$. Bars followed by the same letter(s) are not significantly different $(p \leq 0.05)$. activity against the gram-negative bacteria Klebsiella pneumonia and Gram-positive bacteria S. aureus. However, more reduction was observed in healthy children compared to that of those with ADHD. To the best of our knowledge, this is the first report about the effect of NPs on the number of gut bacteria from healthy and ADHD children by incubating fecal sample on the NA medium.

\section{Changes in Number of Gut Bacteria by Live-Dead Staining}

This result from microscopic observation indicated a lot of live (fluoresce green) and dead (fluoresce red) bacteria in fresh feces from healthy children in the absence of $\mathrm{ZnONP}$. However, the addition of $\mathrm{ZnONP}$ at the concentration of $20 \mu \mathrm{g} / \mathrm{ml}$ caused a marked reduction in the cell numbers of live bacteria in fresh feces from healthy children. In addition, compared to the healthy children, the cell number of live and dead gut bacteria in fresh feces from ADHD children was markedly reduced regardless of the presence or absence of $\mathrm{ZnONP}$ (Figure 4). This result from staining of the live/dead bacterial cells indicates that the ZnONP has a greater inhibition in the growth of gut bacteria in healthy children compared to that of ADHD children, which is consistent with the result of a bacterial number using the plate counting method. However, no more dead cells were observed in the presence of $\mathrm{ZnONP}$
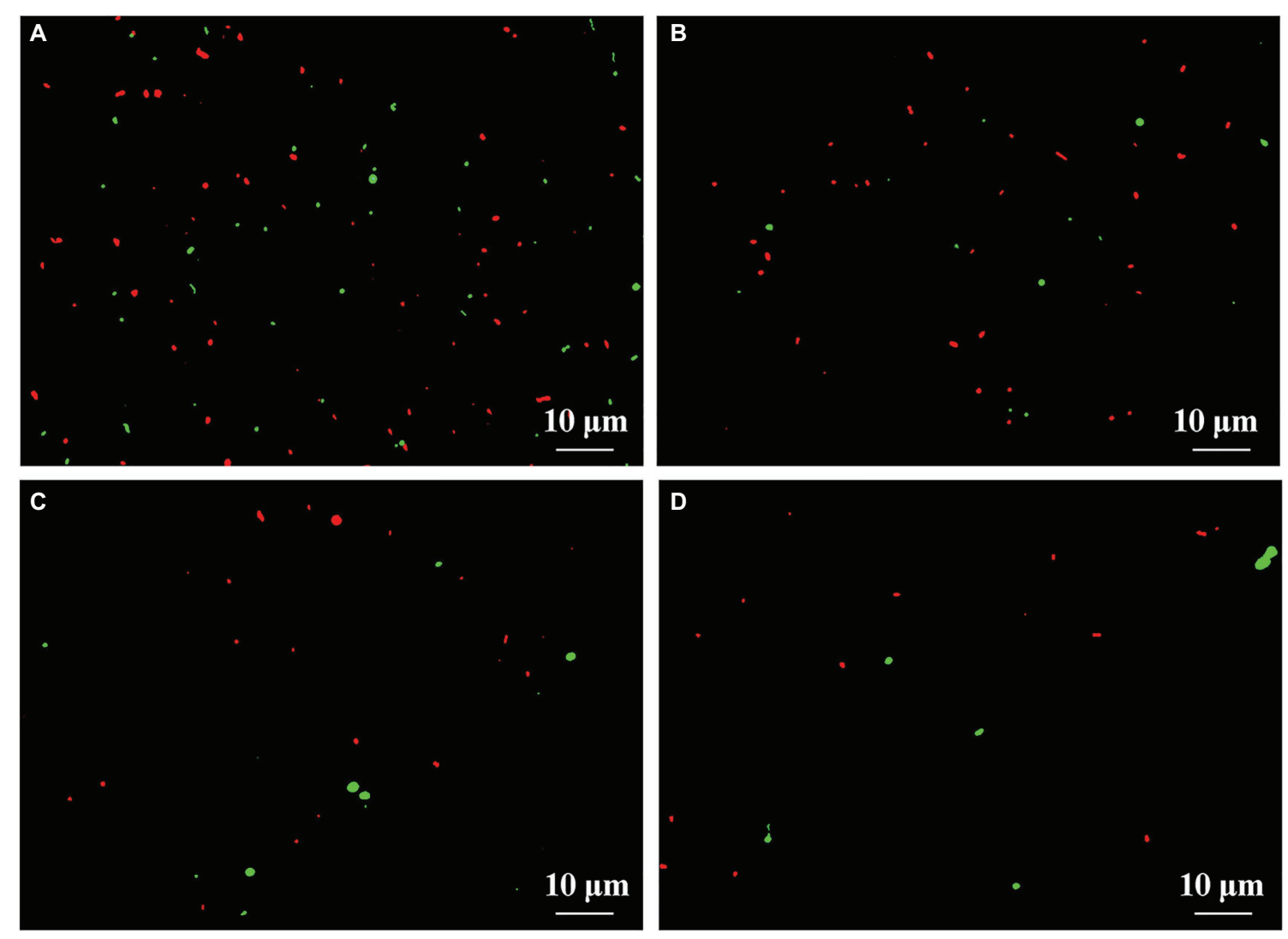

FIGURE 4 | Effect of ZnONP on number of gut bacteria from healthy and ADHD children by live-dead staining. (A) Healthy; (B) healthy + ZnONP; (C) ADHD; and (D) $A D H D+Z n O N P$. 
regardless of healthy or ADHD children, this indicates that the reduction in the number of live cells by this NP may be mainly attributed to its strong inhibition in bacterial replication but not killing bacteria. In contrast with the result of our study, several of our previous studies have revealed that NPs have a bacteriostatic rather than bactericide effect on various bacteria (Masum et al., 2019; Ahmed et al., 2021a).

\section{Changes in Number of Gut Bacteria by Flow Cytometry Observation}

The effect of ADHD and ZnONP on gut bacteria was further determined by comparing the ratio of live with dead gut bacteria in fresh feces from healthy and ADHD children in the presence or absence of $\mathrm{ZnONP}$ calculated by using flow cytometry observation. The results of this study indicated that the percentage of live and dead gut bacteria in healthy children was 56.09 and $43.91 \%$, respectively, while the percentage of live and dead gut bacteria in healthy children was 39.61 and $60.39 \%$, respectively, in the presence of $\mathrm{ZnONP}$. Furthermore, the percentage of live and dead gut bacteria in ADHD children was 58.21 and $41.79 \%$, respectively. In comparison, the percentage of live and dead gut bacteria in ADHD children was 41.85 and $58.15 \%$, respectively, in the presence of $\mathrm{ZnONP}$ (Figure 5). This study indicated a similar ratio of live to dead gut bacteria in fresh feces between healthy and ADHD children in the absence of ZnONP. However, a greater number of live bacteria was observed in the former compared to the latter. However, the percentage of dead gut bacteria was greatly increased by $\mathrm{ZnONP}$ regardless of healthy or ADHA children. Indeed, the addition of $\mathrm{ZnONP}$ caused a $37.53 \%$ increase in the ratio of dead gut bacteria in feces from healthy children, while the addition of $\mathrm{ZnONP}$ caused a $39.15 \%$ increase in the percentage of deadly gut bacteria in the feces from ADHD children. This result indicated that the antibacterial activity of ZnONP may be partially attributed to its bactericidal ability, which is a little different from the
A

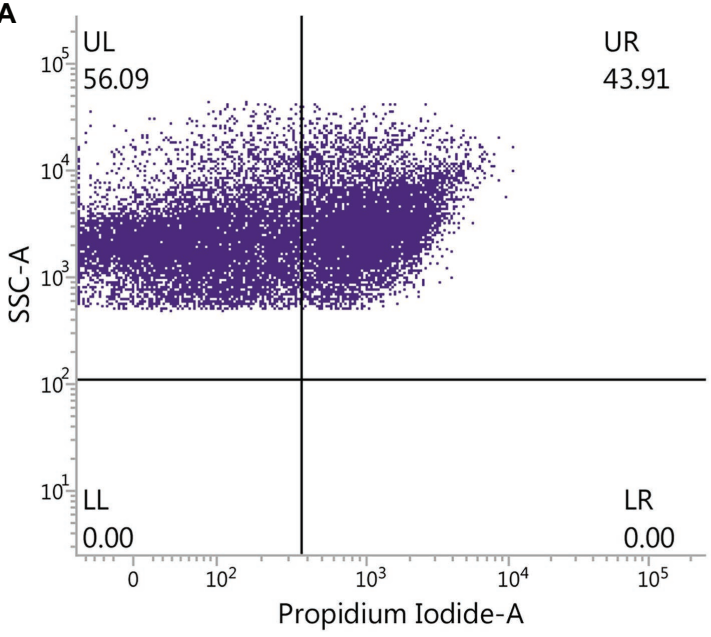

C

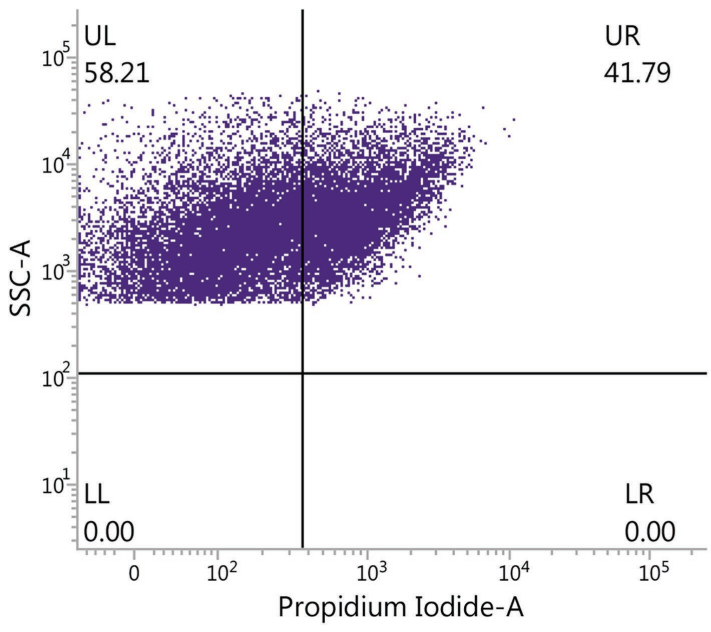

B

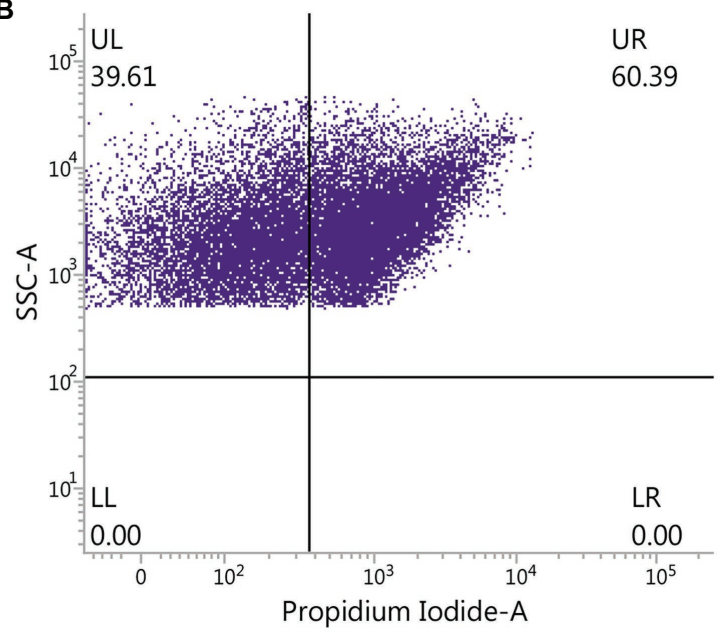

D

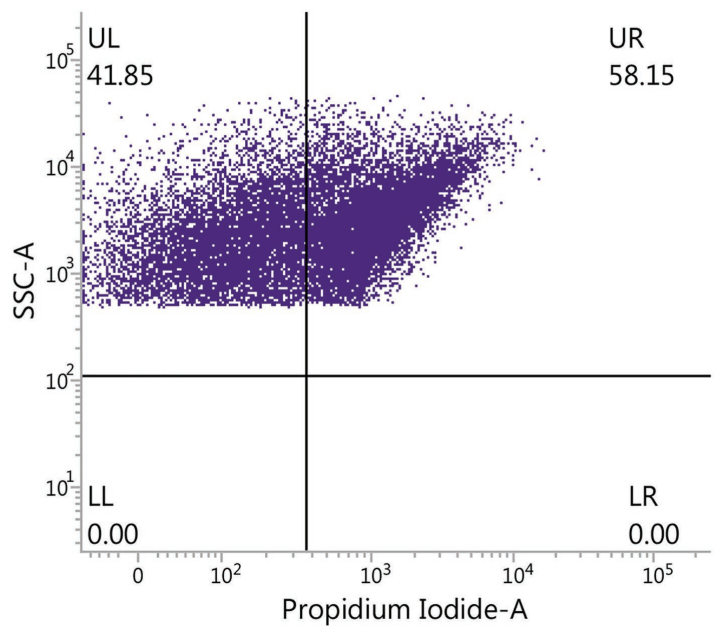

FIGURE 5 | Effect of ZnONP on number of gut bacteria from healthy and ADHD children by flow cytometry observation. (A) Healthy; (B) healthy + ZnONP; (C) ADHD; and (D) ADHD + ZnONP. 
result of the live/dead bacterial cell staining, indicating that the former may have a higher resolution in the antibacterial activity than the latter. Interestingly, these results suggested

TABLE 1 | Sequence similarity of the main representative strains from the fecal samples of the healthy and ADHD children.

\begin{tabular}{|c|c|c|c|}
\hline $\begin{array}{l}\text { Bacterial } \\
\text { strains }\end{array}$ & Sources & $\begin{array}{l}\text { Bacterial identity } \\
\text { (sequence } \\
\text { similarity of } 16 \mathrm{~S} \\
\text { rDNA) }\end{array}$ & Accession no. \\
\hline Strain 2-1 & Healthy + ZnONP & $\begin{array}{l}\text { Escherichia } \\
\text { fergusonii (99.79\%) }\end{array}$ & MW664943 \\
\hline Strain 2-2 & Healthy + ZnONP & $\begin{array}{l}\text { Bacillus cereus } \\
(99.12 \%)\end{array}$ & MW664944 \\
\hline Strain 2-3 & Healthy + ZnONP & $\begin{array}{l}\text { Escherichia coli } \\
(99.58 \%)\end{array}$ & MW664945 \\
\hline Strain 2-4 & Healthy + ZnONP & $\begin{array}{l}\text { Escherichia sp. } \\
(99.38 \%)\end{array}$ & MW664946 \\
\hline Strain 4-1 & $\mathrm{ADHD}+\mathrm{ZnONP}$ & $\begin{array}{l}\text { Bacillus thuringiensis } \\
(99.06 \%)\end{array}$ & MW664947 \\
\hline Strain 4-2 & $\mathrm{ADHD}+\mathrm{ZnONP}$ & $\begin{array}{l}\text { Bacillus } \\
\text { paramycoides } \\
(98.80 \%)\end{array}$ & MW664948 \\
\hline Strain 4-3 & $\mathrm{ADHD}+\mathrm{ZnONP}$ & $\begin{array}{l}\text { Bacillus anthracis } \\
(99.69 \%)\end{array}$ & MW664949 \\
\hline
\end{tabular}

that the reduction in number of gut bacteria is mainly due to $\mathrm{ADHD}$ other than $\mathrm{ZnONP}$, which caused a similar increase between healthy and ADHD children in the percentage of dead cells.

\section{Identification of Gut Bacteria Resistant to NPs}

Several researchers have tried to investigate the population of the general gut microbiota in ADHD children using the nonculture method (Stevens et al., 2019; Wang et al., 2020), which gives a comprehensive analysis in the composition of gut bacteria from ADHD children. Indeed, the nonculture method has the advantage of quicker results, less sample, and less cost, but it also has the disadvantage of not being able to know whether these gut bacteria is live or dead. In contrast, the culture method in this study provides an alternate strategy to the analysis of the gut microbiota profiles by isolating the gut bacteria and then focusing on the live bacteria in children with ADHD. In order to clarify the main bacterial species that resistant to NPs, the main representative bacterial colonies. The collected colonies from the healthy children were generally classified into four different groups, while the colonies collected from the ADHD children were classified into three different groups. The identity of each group was further determined
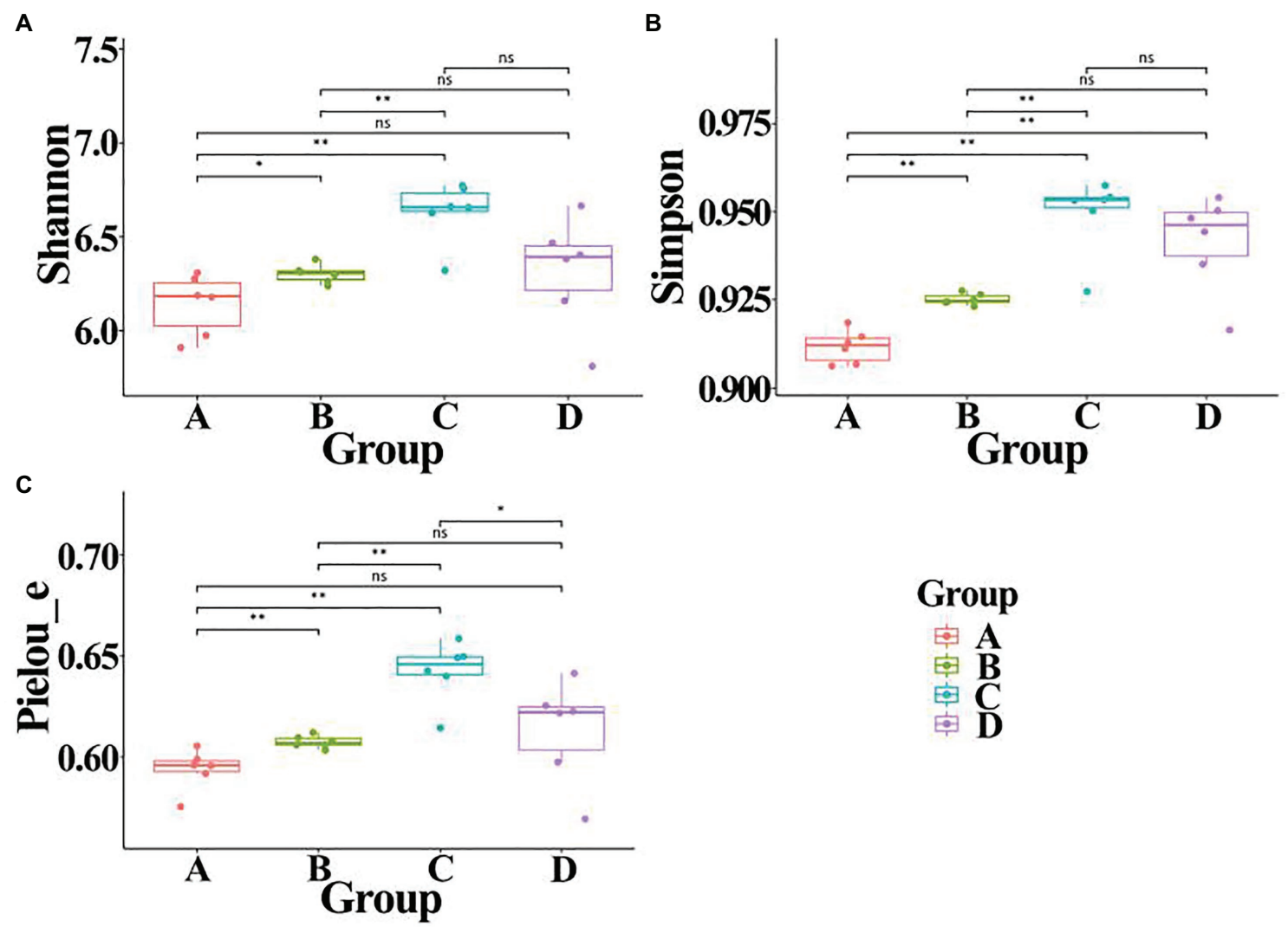

FIGURE 6 | Alpha diversity of gut bacteria from healthy and ADHD children in the presence or absence of ZnONP. (A) Shannon index; (B) Simpson index; and (C) Pielou_e index. A: Healthy; B: healthy + ZnONP; C: ADHD; and D: ADHD + ZnONP. Vertical bars represent standard errors of the mean $(n=6)$. ${ }^{*} p \leq 0.05$; ${ }^{* *} p \leq 0.01$. ns, not significant. 


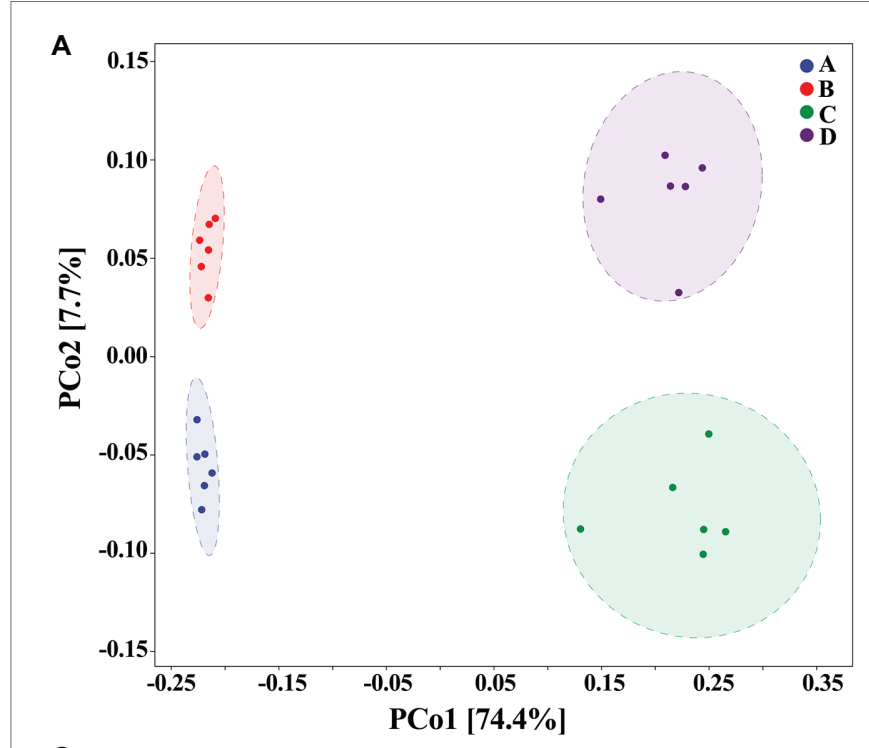

C

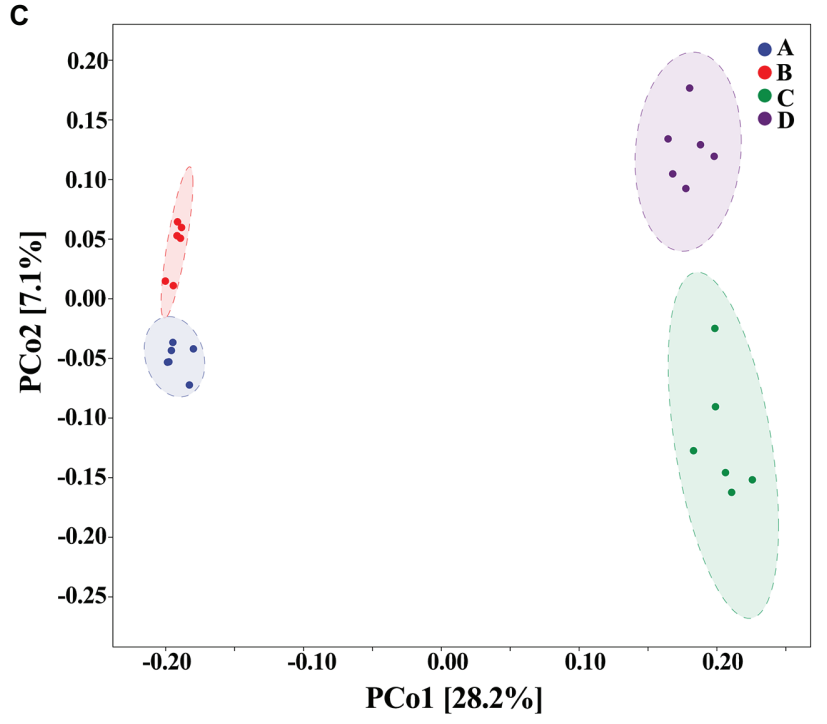

B

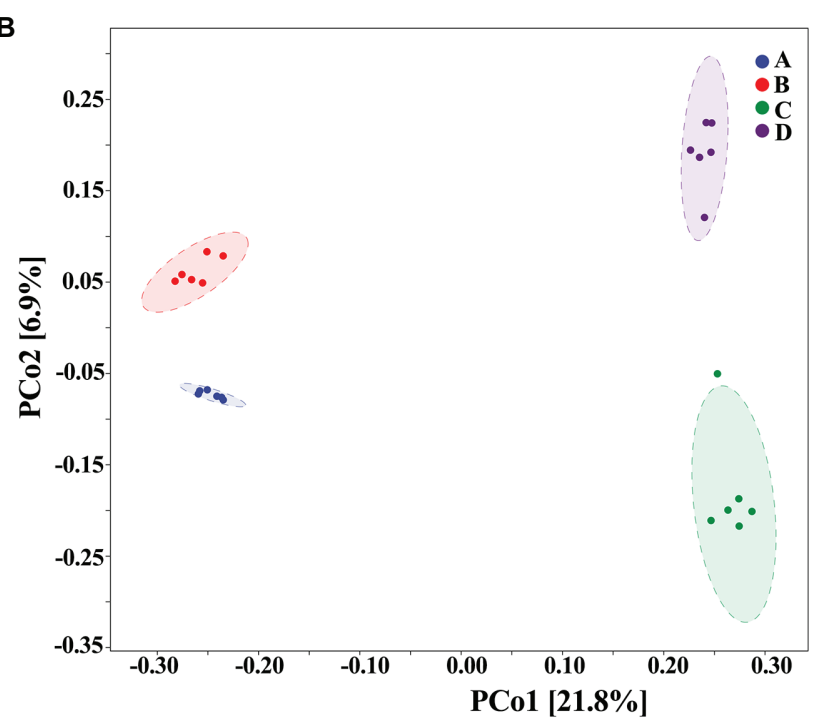

D

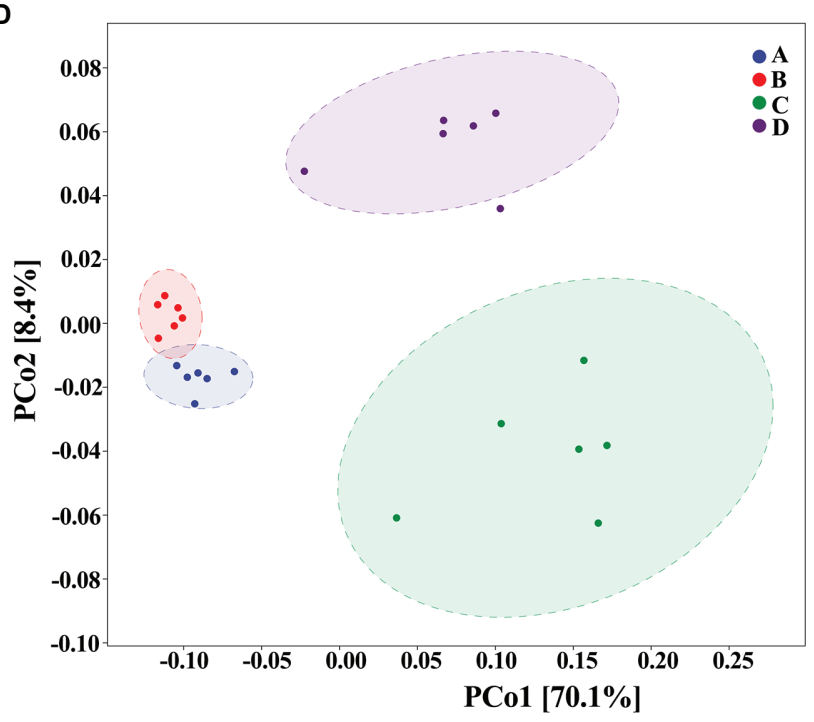

FIGURE 7 | Beta diversity of gut bacteria by principal coordinates analysis (PCoA; $n=6$ ) of (A) Bray-Curtis, (B) Jaccard, (C) unweighted UniFrac, and (D) weighted UniFrac. A: Healthy; B: healthy + ZnONP; C: ADHD; and D: ADHD + ZnONP.

based on the analysis of $16 \mathrm{~S}$ rDNA sequence of the representative isolates, which were collected by picking out from the NA medium supplement with ZnONP of $20 \mu \mathrm{g} / \mathrm{ml}$ based on the shape, color, and edge of colony. Indeed, sequence analysis of representative colonies (strains 2-1, 2-2, 2-3, and 2-4) indicated that Escherichia fergusonii, B. cereus, E. coli, and Escherichia sp. were the main bacterial species in fresh feces from healthy children, while sequence analysis of representative colonies (strains 4-1, 4-2, and 4-3) indicated that B. cereus, Bacillus paramycoides, and Bacillus anthracis were the main bacterial species in fresh feces from ADHD children (Table 1). Results of this study indicated that the main gut bacterial community resistant to NPs in fecal samples from healthy children was greatly different from that from ADHD children. The main NP-resistant gut bacteria in healthy children belongs to the genus Escherichia, while the main NP-resistant gut bacteria in healthy children belongs to the genus Bacillus. This may be mainly due to the difference in community structure between healthy and ADHD children. In agreement with the result of our study, the change of community structure in ADHD children has been reported in many previous studies (Stevens et al., 2019; Wang et al., 2020). Interestingly, the result clearly indicated that some gut bacteria show great resistance to NPs, which may be used to adjust the community structure of gut bacteria by combining NPs.

\section{Community Diversity Determined by $16 \mathrm{~S}$ rRNA Amplicon Sequencing}

In order to calculate alpha diversity of gut bacteria, data of each sample were normalized to the same number of reads (33,680 sequences, the smallest number of sequences). A comprehensive analysis was carried out in this study by calculating 


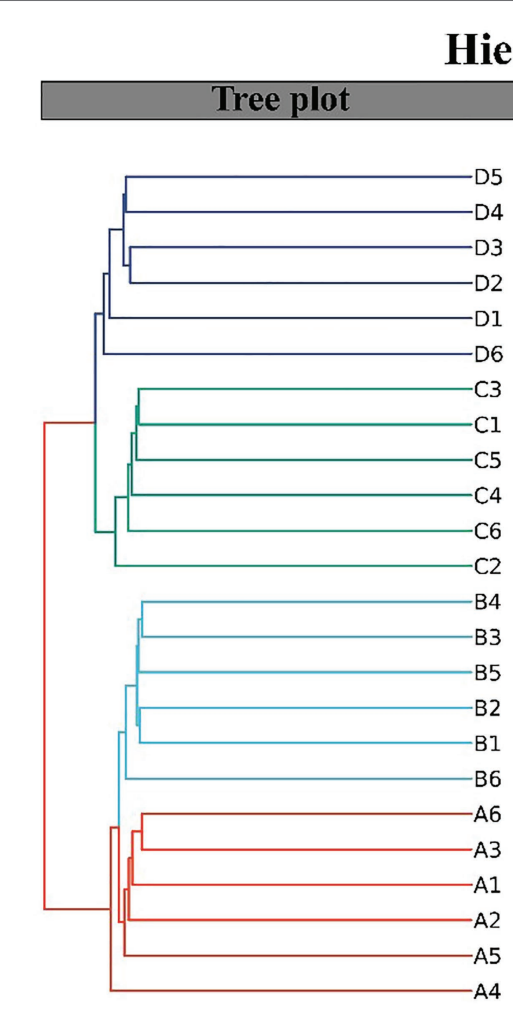

Hierarchical clustering analysis
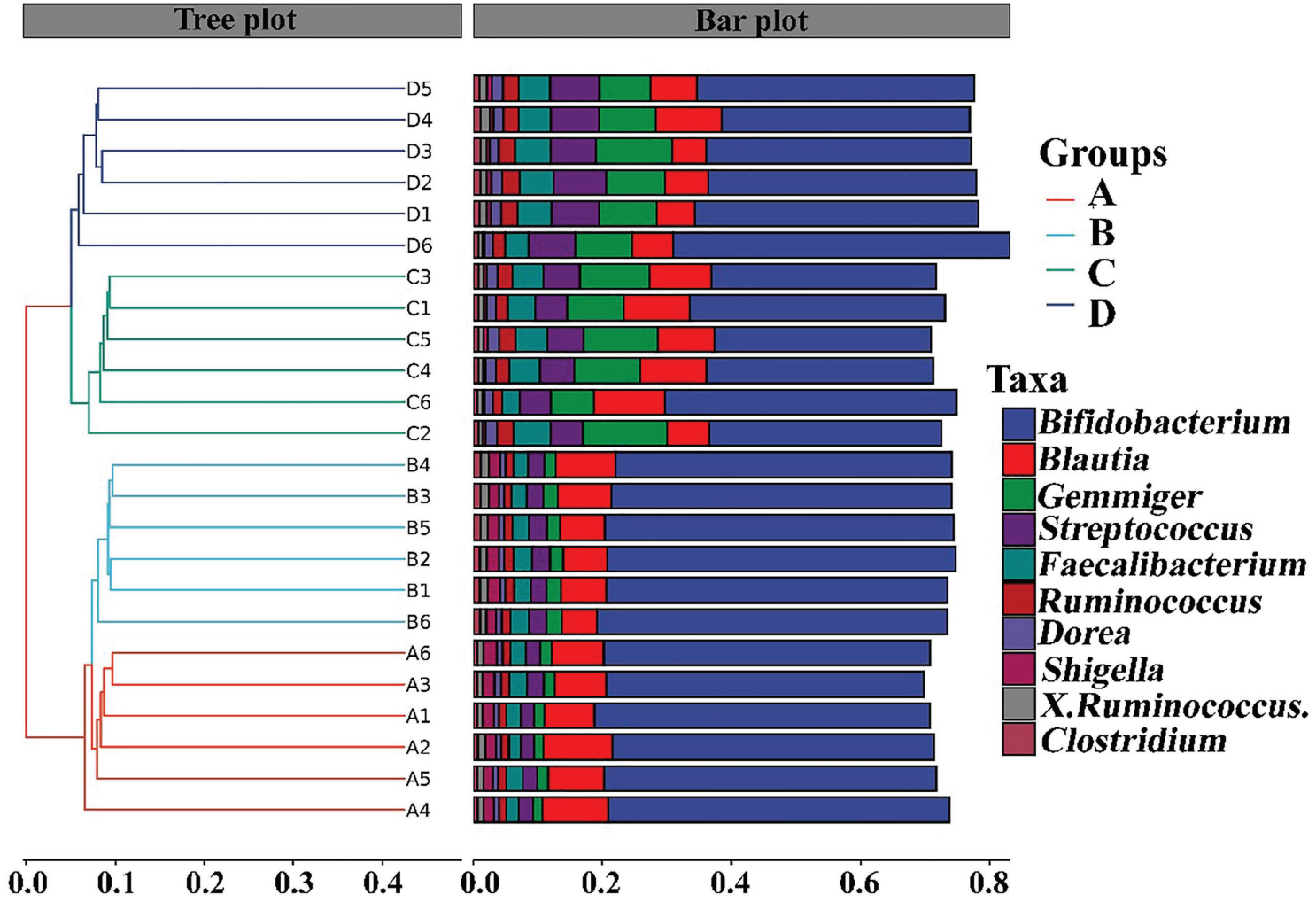

FIGURE 8 | Analysis of hierarchical clustering constructed at the genus level by Bray-Curtis dissimilarity $(n=6)$. A: Healthy; B: healthy + ZnONP; C: ADHD; and D: $A D H D+Z n O N P$.

Shannon, Simpson, and Pielou_e indexes. Result of Figure 6 indicated that the community diversity index of gut bacteria from ADHD children was significantly higher in the three different indexes than that of healthy children $(p<0.01$; Figure 3). This is different with the result of Aarts et al. (2017), who found that there was no difference in alpha diversity of gut microbiota between patients with ADHD and controls using 16S rRNA marker gene sequencing. However, in agreement with the data of our study, Wang et al. (2020) found that the gut microbiota communities in ADHD patients showed a significantly higher Shannon index than the control subjects by using 16S rRNA V3V4 amplicon sequencing.

On the other hand, our results also showed that the biosynthesized NPs had a differential effect between healthy and ADHD children in the community diversity index of gut bacteria. Indeed, the addition of $\mathrm{ZnONP}$ caused a significant $(p<0.05$ or 0.01$)$ increase in the three different community diversity index of gut bacteria from healthy children. In contrast, the Pielou_e index was significantly $(p<0.05)$ reduced, while Shannon and Simpson indexes of gut bacteria from ADHD children were unaffected by $\mathrm{ZnONP}$ (Figure 6). Thus, it can be inferred from this result that there was a difference in the community structure of gut bacteria between healthy and ADHD children. The beta diversity of gut bacteria in fecal samples from healthy and
ADHD children in the presence or absence of $\mathrm{ZnONP}$ was further analyzed by using PCoA (principal coordinates analysis) of Bray-Curtis, Jaccard, unweighted UniFrac, and weighted UniFrac at the ASVS level. In general, PCoA analysis of the four different indicators showed a 3.5-74.4\% variation of bacterial communities among these fecal samples. In detail, PC1 explained $74.4,21.8,28.2$, and $70.1 \%$ of the variation, respectively, between healthy and ADHD children in Bray-Curtis, Jaccard, unweighted UniFrac, and weighted UniFrac parameters, while PC2 explained 7.7, 6.9, 7.1, and $3.5 \%$ of the variation, respectively, between with and without NPs in the above-mentioned four different indicators (Figure 7).

In contrast with the result, Wang et al. (2020) found that the gut microbiomes were similar between the healthy controls and ADHD patients based on the unweighted UniFrac and weighted UniFrac plots. It can be inferred from these results that there was obvious differences in gut bacterial communities among the tested samples, which could be effectively differentiated by the above-mentioned four different indicators (Figure 7). However, the differential effect depends on the four selected parameters and the two principle components. Indeed, ADHD showed a greater differential effect than that of NP. In agreement with the result of diversity analysis, we also found that ADHD has a greater influence in change of gut bacterial communities 


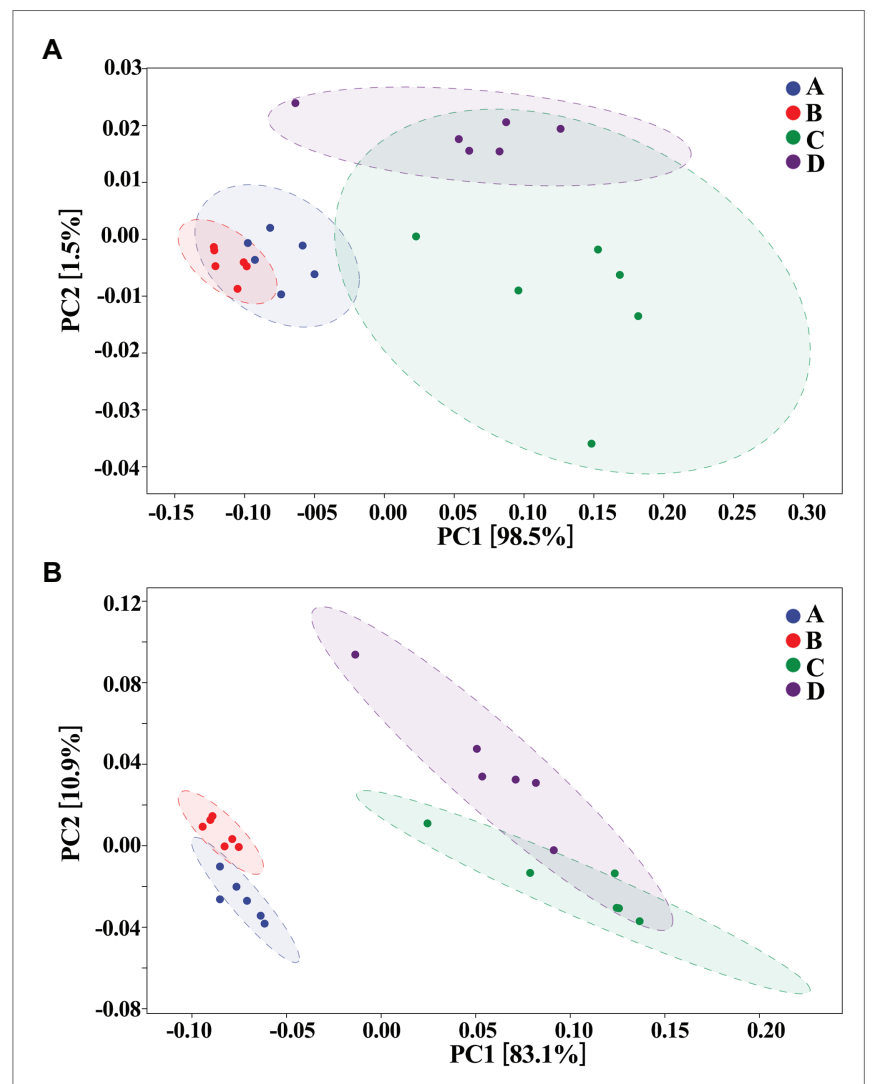

C

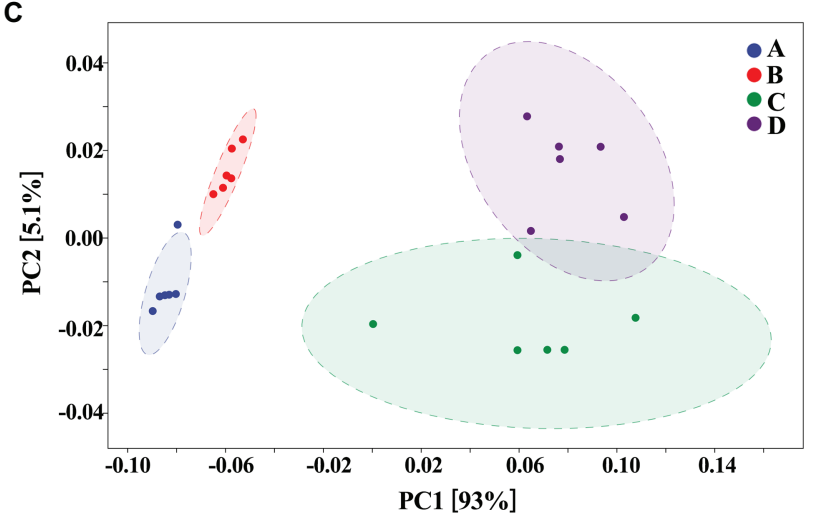

FIGURE 9 | Principal components analysis (PCA; $n=6$ ) at (A) phylum, (B) genus, and (C) species levels. A: Healthy; B: healthy + ZnONP; C: ADHD; and $\mathrm{D}: \mathrm{ADHD}+\mathrm{ZnONP}$.

than NPs based on the analysis of hierarchical clustering, which was constructed at the genus level by Bray-Curtis dissimilarity (Figure 8).

\section{Species Composition Determined by $16 S$ rRNA Amplicon Sequencing}

The composition of gut bacteria was determined from six replicates of each samples by PCA of phylum, genus, and species, which showed that there was an overall similarity in bacterial community structure within each sample. However, the community structure differed in different samples. Indeed, the result of PCA showed that there was a 95.6, 83.1, and 93\% total variation between healthy and ADHD children in PC1 at the phylum, genus, and species levels, respectively, while there was a $3.5,10.9$, and $5.1 \%$ total variation between with and without NPs in PC2 at the phylum, genus, and species levels, respectively (Figure 9). This result consists of the data of diversity analysis, which indicated that ADHA achieved a greater change of gut bacterial communities than NPs. Following the comparison with the greengene database, we found that the top eight phylum of gut bacteria from these samples were Actinobacteria, Firmicutes, Proteobacteria, Bacteroide, Verrucomicrobia, TM7, Tenericutes, and Fusobacteria, of which Actinobacteria (49.6\%) and Firmicutes (46.8\%) were the main groups. At the genus level, there was no difference in the taxonomic composition between healthy children in presence and absence of NPs, as well as between ADHD children in presence and absence of NPs, but there was a difference between healthy and ADHD children. For example, the relative abundances of Bifidobacterium and Gemmiger in healthy children NPs were 52.1 and $9.7 \%$, respectively; in contrast, the two genus in ADHD children were 40.3 and $1.8 \%$, respectively, regardless of the presence or absence of NPs (Figure 10).

In order to further compare the differences in species composition between the samples and display the trend of species abundance distribution of each sample, the heat map was draw by selecting the abundance data of the top 20 genera with average abundance. As shown in Figure 11, the results of this study revealed a significant change in the intestinal microbial community structure between healthy and ADHD children, which is in agreement with the data of previous reports (Stevens et al., 2019; Wang et al., 2020). Indeed, Shigella, SMB53, Turicibacter, Shigella, Bifidobacterium, Collinsella, Ruminococcus, and Clostridium are enriched in healthy children regardless of the presence or absence of NPs, while Roseburia, Gemmiger, Acinetobacter, Enterococcus, Bacteroides, Streptococcus, and Faecalibacterium were enriched in ADHD children regardless of the presence or absence of NPs. However, different bacterial species were changed because of Wang et al. (2020), who revealed that the relative abundance of Bacteroides coprocola was decreased, while the relative abundance of Bacteroides uniformis, Bacteroides ovatus, and Sutterella stercoricanis were increased in the ADHD group.

Undoubtedly, determination of ADHD-associated gut bacteria will open the possibility for new potential intervention strategies in the prevention of this disease; however, the conflicted results were often found in different studies in this field; for example, although the correlation of the genus Bifidobacterium with ADHD symptoms has been identified in several recent studies, the outcomes of Bifidobacterium in ADHD is still controversial. Several studies found that Bifidobacterium has a protective effect against ADHD, whereas Aarts et al. (2017) reported that ADHD patients had a 12.7-20.5 increased Bifidobacterium genus to controls. This may be mainly due to the difference in the objective and method of study. In general, this study indicated that $\mathrm{ADHD}$ caused the reduction in number of gut bacteria but increased the diversity of community, suggesting that the diversity of microbiome community may be related 


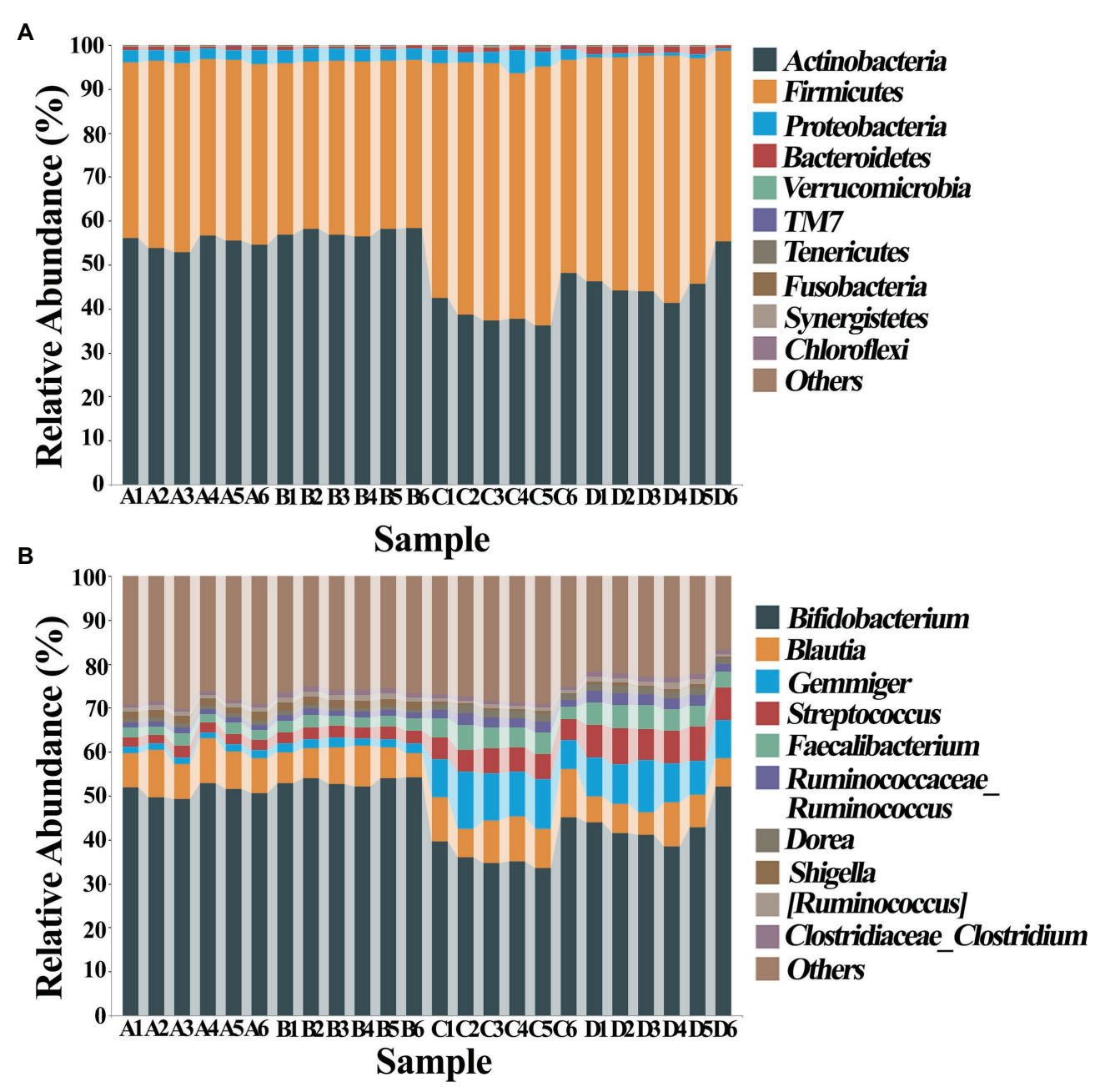

FIGURE 10 | Classification and composition diagram $(n=6)$ at $(\mathbf{A})$ phylum and $(\mathbf{B})$ genus levels. A: Healthy; B: healthy + ZnONP; C: ADHD; and D: $A D H D+Z n O N P$.

to the development of ADHD. Interestingly, the change of gut bacteria in ADHD is similar to that in ASD (autism spectrum disorder, another children disease) children in our previous studies (Yu et al., 2013, 2020). This can be justified by the result of many studies, which have found that comorbidity is high in ASD and ADHD individuals (Bölte et al., 2014). Fortunately, our results clearly indicated that NPs can decrease the diversity of microbiome community in ADHD children. Furthermore, some NP-resistant bacteria were successfully isolated, purified, and identified in this study. Therefore, it can be inferred that NPs and its resistant strains may potentially modulate and regulate the gut microbiome community of ADHD children.

\section{CONCLUSION}

In this study, we have biologically synthesized and characterized the ZnONPs by using bacterial strain RTN1. Moreover, we investigated the impact of biogenic ZnONPs on the gut microbiota of healthy and ADHD children in China based on the traditional plate method and amplicon sequencing analysis for the first time. Compared to healthy children, significant changes were noted in the number and composition of gut bacteria from the fecal samples of children with ADHD, indicating these bacteria residing in the gut may contribute to ADHD development. In addition, we found that NPs have a differential effect in composition of gut bacteria from healthy and ADHD children, while the NP-resistant strains in healthy children are also different from that in ADHD children. Overall, the result not only revealed the potential correlation of ADHD with gut bacteria but also found that biogenic $\mathrm{ZnONPs}$ could change the number and composition of gut bacteria. In particular, some gut bacteria that are resistant to biogenic ZnONPs have been successfully isolated and identified, which gave a new strategy to prevent ADHD by the combination of NP and its resistant bacteria. However, future studies are also needed to assess the effect of ZnONPs on the normal cells and beneficial microbiota, as well as animal trials for their large-scale applications. 


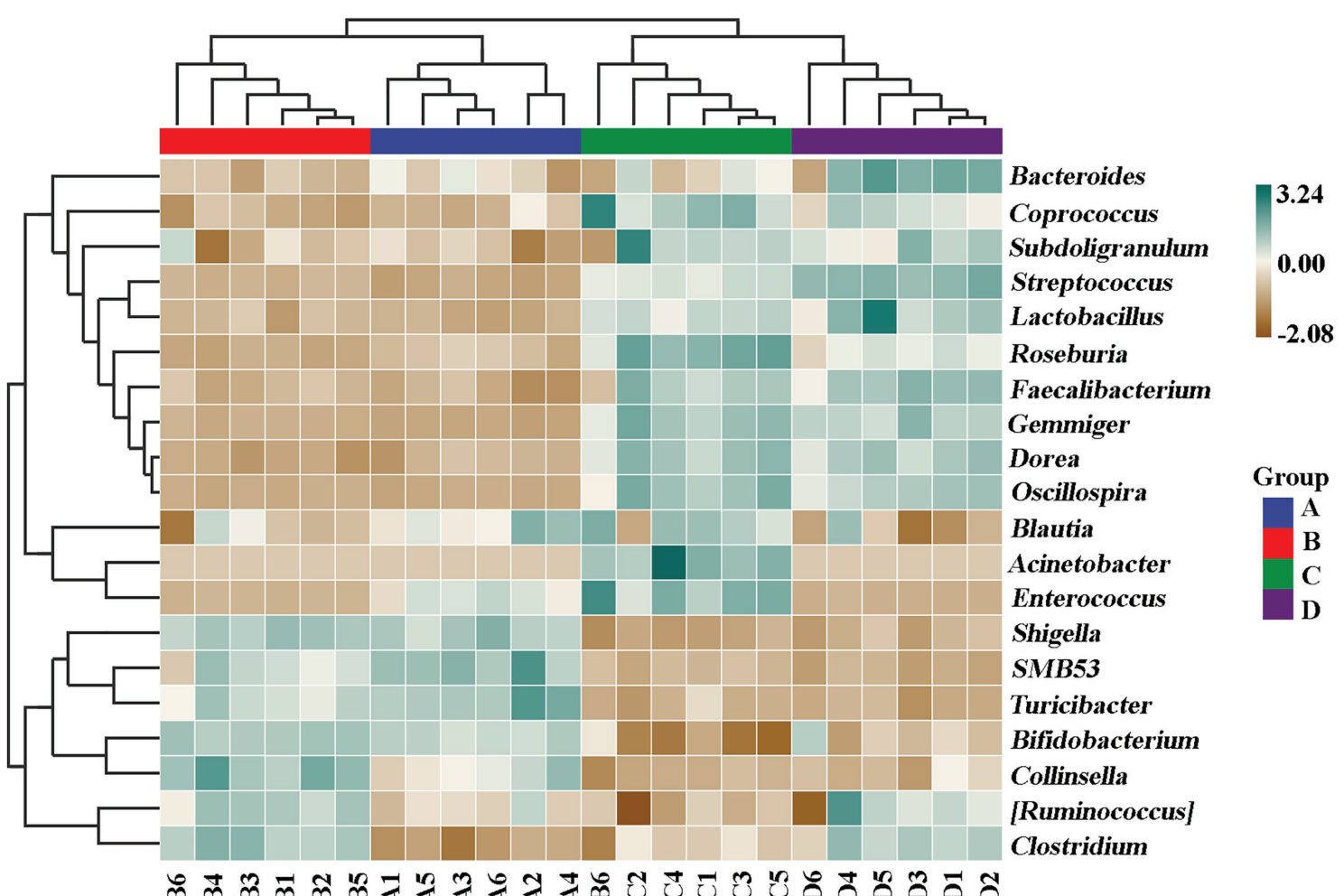

FIGURE 11 | Composition and distribution of the main 20 genus among samples $(n=6)$.

\section{DATA AVAILABILITY STATEMENT}

The datasets presented in this study can be found in online repositories. The names of the repository/repositories and accession number(s) can be found at: https://www.ncbi.nlm. nih.gov/, MW664943, MW664944, MW664945, MW664946, MW664947, MW664948, and MW664949.

\section{AUTHOR CONTRIBUTIONS}

GZ, RY, and BL: conceptualization. GZ, RY, HJ, MZ, LL, TA, and BL: methodology and writing - original draft. RY, HJ,

\section{REFERENCES}

Aarts, E., Ederveen, T. H., Naaijen, J., Zwiers, M. P., Boekhorst, J., Timmerman, H. M., et al. (2017). Gut microbiome in ADHD and its relation to neural reward anticipation. PLoS One 12:e183509. doi: 10.1371/journal. pone.0183509

Abid, M. A., Kadhim, D. A., and Aziz, W. J. (2020). Iron oxide nanoparticle synthesis using trigonella and tomato extracts and their antibacterial activity. Mater. Technol. 25, 1-8. doi: 10.1080/10667857.2020.1863572

Agarwal, H., Menon, S., Kumar, S. V., and Rajeshkumar, S. (2018). Mechanistic study on antibacterial action of zinc oxide nanoparticles synthesized using green route. Chem. Biol. Interact. 286, 60-70. doi: 10.1016/j.cbi.2018.03.008

Ahmed, T., Noman, M., Luo, J., Muhammad, S., Shahid, M., Ali, M. A., et al. (2021a). Bioengineered chitosan-magnesium nanocomposite: a novel agricultural antimicrobial agent against Acidovorax oryzae and Rhizoctonia
LL, and TA: software. GZ, FA, KA, and RY: data investigation. BL: supervision. KA, GZ, RY, and BL: visualization. GZ, RY, TA, HJ, MZ, LL, FA, KA, and BL: writing - review and editing. All authors contributed to the article and approved the submitted version.

\section{FUNDING}

This research was funded by the Science and Technology Project of Zhejiang Provincial Health Commission, China (2019KY517) and Project of Hangzhou Science and Technology Commission (20191203B121).

solani for sustainable rice production. Int. J. Biol. Macromol. 168, 834-845. doi: 10.1016/j.ijbiomac.2020.11.148

Ahmed, T., Noman, M., Shahid, M., Shahid, M. S., and Li, B. (2021b). Antibacterial potential of green magnesium oxide nanoparticles against rice pathogen Acidovorax oryzae. Mater. Lett. 282:128839. doi: 10.1016/j.matlet.2020.128839

Ahmed, T., Shahid, M., Noman, M., Niazi, M. B. K., Zubair, M., Almatroudi, A., et al. (2020). Bioprospecting a native silver-resistant Bacillus safensis strain for green synthesis and subsequent antibacterial and anticancer activities of silver nanoparticles. J. Adv. Res. 24, 475-483. doi: 10.1016/j. jare.2020.05.011

Ahmed, T., Wu, Z., Jiang, H., Luo, J., Noman, M., Shahid, M., et al. (2021c). Bioinspired green synthesis of zinc oxide nanoparticles from a native Bacillus cereus strain RNT6: characterization and antibacterial activity against rice panicle blight pathogens Burkholderia glumae and B. gladioli. Nanomaterials 11:884. doi: 10.3390/nano11040884 
Ali, M., Ahmed, T., Wu, W., Hossain, A., Hafeez, R., Islam Masum, M., et al. (2020). Advancements in plant and microbe-based synthesis of metallic nanoparticles and their antimicrobial activity against plant pathogens. Nanomaterials 10:1146. doi: 10.3390/nano10061146

Alphandéry, E. (2020). Bio-synthesized iron oxide nanoparticles for cancer treatment. Int. J. Pharm. 586:119472. doi: 10.1016/j.ijpharm.2020.119472

Bölte, S., Willfors, C., Berggren, S., Norberg, J., Poltrago, L., Mevel, K., et al. (2014). The roots of autism and ADHD twin study in Sweden (RATSS). Twin Res. Hum. Genet. 17, 164-176. doi: 10.1017/thg.2014.12

Boonchooduang, N., Louthrenoo, O., Chattipakorn, N., and Chattipakorn, S. C. (2020). Possible links between gut-microbiota and attention-deficit/ hyperactivity disorders in children and adolescents. Eur. J. Nutr. 59, 3391-3403. doi: 10.1007/s00394-020-02383-1

Bundgaard-Nielsen, C., Knudsen, J., Leutscher, P. D., Lauritsen, M. B., Nyegaard, M., Hagstrøm, S., et al. (2020). Gut microbiota profiles of autism spectrum disorder and attention deficit/hyperactivity disorder: a systematic literature review. Gut Microbes 11, 1172-1187. doi: 10.1080/19490976.2020.1748258

Callahan, B. J., McMurdie, P. J., Rosen, M. J., Han, A. W., Johnson, A. J. A., and Holmes, S. P. (2016). DADA2: high-resolution sample inference from illumina amplicon data. Nat. Methods 13, 581-583. doi: 10.1038/nmeth.3869

Checa-Ros, A., Jeréz-Calero, A., Molina-Carballo, A., Campoy, C., and Muñoz-Hoyos, A. (2021). Current evidence on the role of the gut microbiome in ADHD pathophysiology and therapeutic implications. Nutrients 13:249. doi: $10.3390 /$ nul3010249

De Vadder, F., Kovatcheva-Datchary, P., Goncalves, D., Vinera, J., Zitoun, C., Duchampt, A., et al. (2014). Microbiota-generated metabolites promote metabolic benefits via gut-brain neural circuits. Cell 156, 84-96. doi: 10.1016/j.cell.2013.12.016

Gericke, M., and Pinches, A. (2006). Biological synthesis of metal nanoparticles. Hydrometallurgy 83, 132-140. doi: 10.1016/j.hydromet.2006.03.019

Hayashi, W., and Iwanami, A. (2018). Biological mechanisms of ADHD. Brain Nerve 70, 1265-1277. doi: 10.11477/mf.1416201172

Hiergeist, A., Gessner, J., and Gessner, A. (2020). Current limitations for the assessment of the role of the gut microbiome for attention deficit hyperactivity disorder (ADHD). Front. Psychiatry 11:623. doi: 10.3389/fpsyt.2020.00623

Karmakar, A., Goswami, R., Saha, T., Maitra, S., Roychowdhury, A., Panda, C. K., et al. (2017). Pilot study indicate role of preferentially transmitted monoamine oxidase gene variants in behavioral problems of male ADHD probands. BMC Med. Genet. 18:109. doi: 10.1186/s12881-017-0469-5

Kummara, S., Patil, M. B., and Uriah, T. (2016). Synthesis, characterization, biocompatible and anticancer activity of green and chemically synthesized silver nanoparticles-a comparative study. Biomed. Pharmacother. 84, 10-21. doi: 10.1016/j.biopha.2016.09.003

Li, B., Wang, X., Chen, R., Huangfu, W., and Xie, G. (2008). Antibacterial activity of chitosan solution against Xanthomonas pathogenic bacteria isolated from Euphorbia pulcherrima. Carbohydr. Polym. 72, 287-292. doi: 10.1016/j. carbpol.2007.08.012

Luo, Y., Weibman, D., Halperin, J. M., and Li, X. (2019). A review of heterogeneity in attention deficit/hyperactivity disorder (ADHD). Front. Hum. Neurosci. 13:42. doi: $10.3389 /$ fnhum.2019.00042

Malaikozhundan, B., Vaseeharan, B., Vijayakumar, S., and Thangaraj, M. P. (2017). Bacillus thuringiensis coated zinc oxide nanoparticle and its biopesticidal effects on the pulse beetle, Callosobruchus maculatus. J. Photochem. Photobiol. B 174, 306-314. doi: 10.1016/j.jphotobiol.2017.08.014

Martins-Silva, T., Salatino-Oliveira, A., Genro, J. P., Meyer, F. D., Li, Y., Rohde, L. A., et al. (2021). Host genetics influences the relationship between the gut microbiome and psychiatric disorders. Prog. Neuro Psychopharmacol. Biol. Psychiatry 106:110153. doi: 10.1016/j.pnpbp.2020.110153

Masum, M., Islam, M., Siddiqa, M., Ali, K. A., Zhang, Y., Abdallah, Y., et al. (2019). Biogenic synthesis of silver nanoparticles using Phyllanthus emblica fruit extract and its inhibitory action against the pathogen Acidovorax oryzae strain RS-2 of rice bacterial brown stripe. Front. Microbiol. 10:820. doi: 10.3389/fmicb.2019.00820

Ogunyemi, S. O., Abdallah, Y., Zhang, M., Fouad, H., Hong, X., Ibrahim, E., et al. (2019a). Green synthesis of zinc oxide nanoparticles using different plant extracts and their antibacterial activity against Xanthomonas oryzae pv. oryzae. Artif. Cells Nanomed. Biotechnol. 47, 341-352. doi: 10.1080/21691401.2018.1557671

Ogunyemi, S. O., Zhang, M., Abdallah, Y., Ahmed, T., Qiu, W., Ali, M., et al. (2020). The bio-synthesis of three metal oxide nanoparticles $\left(\mathrm{ZnO}, \mathrm{MnO}_{2}\right.$, and $\mathrm{MgO}$ ) and their antibacterial activity against the bacterial leaf blight pathogen. Front. Microbiol. 11:588326. doi: 10.3389/fmicb.2020.588326

Ogunyemi, S. O., Zhang, F., Abdallah, Y., Zhang, M., Wang, Y., Sun, G., et al. (2019b). Biosynthesis and characterization of magnesium oxide and manganese dioxide nanoparticles using Matricaria chamomilla L. extract and its inhibitory effect on Acidovorax oryzae strain RS-2. Artif. Cells Nanomed. Biotechnol. 47, 2230-2239. doi: 10.1080/21691401.2019.1622552

Pietroiusti, A., Magrini, A., and Campagnolo, L. (2016). New frontiers in nanotoxicology: gut microbiota/microbiome-mediated effects of engineered nanomaterials. Toxicol. Appl. Pharmacol. 299, 90-95. doi: 10.1016/j. taap.2015.12.017

Sampson, T. R., and Mazmanian, S. K. (2015). Control of brain development, function, and behavior by the microbiome. Cell Host Microbe 17, 565-576. doi: $10.1016 /$ j.chom.2015.04.011

Saravanan, M., Gopinath, V., Chaurasia, M. K., Syed, A., Ameen, F., and Purushothaman, N. (2018). Green synthesis of anisotropic zinc oxide nanoparticles with antibacterial and cytofriendly properties. Microb. Pathog. 115, 57-63. doi: 10.1016/j.micpath.2017.12.039

Selvarajan, E., and Mohanasrinivasan, V. (2013). Biosynthesis and characterization of $\mathrm{ZnO}$ nanoparticles using Lactobacillus plantarum VITES07. Mater. Lett. 112, 180-182. doi: 10.1016/j.matlet.2013.09.020

Stevens, A. J., Purcell, R. V., Darling, K. A., Eggleston, M. J., Kennedy, M. A., and Rucklidge, J. J. (2019). Human gut microbiome changes during a 10 week randomised control trial for micronutrient supplementation in children with attention deficit hyperactivity disorder. Sci. Rep. 9:10128. doi: 10.1038/ s41598-019-46146-3

Suresh, J., Pradheesh, G., Alexramani, V., Sundrarajan, M., and Hong, S. I. (2018). Green synthesis and characterization of zinc oxide nanoparticle using insulin plant (Costus pictus D. Don) and investigation of its antimicrobial as well as anticancer activities. Adv. Nat. Sci. Nanosci. Nanotechnol. 9:15008. doi: 10.1088/2043-6254/aaa6fl

Szopinska-Tokov, J., Dam, S., Naaijen, J., Konstanti, P., Rommelse, N., Belzer, C., et al. (2020). Investigating the gut microbiota composition of individuals with attention-deficit/hyperactivity disorder and association with symptoms. Microorganisms 8:406. doi: 10.3390/microorganisms8030406

Wan, L., Ge, W.-R., Zhang, S., Sun, Y.-L., Wang, B., and Yang, G. (2020). Case-control study of the effects of gut microbiota composition on neurotransmitter metabolic pathways in children with attention deficit hyperactivity disorder. Front. Neurosci. 14:127. doi: 10.3389/fnins.2020.00127

Wang, L.-J., Yang, C.-Y., Chou, W.-J., Lee, M.-J., Chou, M.-C., Kuo, H.-C., et al. (2020). Gut microbiota and dietary patterns in children with attentiondeficit/hyperactivity disorder. Eur. Child Adolesc. Psychiatry 29, 287-297. doi: 10.1007/s00787-019-01352-2

Yu, R., Wu, Z., Wang, S., Zhang, M., Zhou, G., and Li, B. (2020). Isolation, identification and characterization of propionic acid bacteria associated with autistic spectrum disorder. Microb. Pathog. 147:104371. doi: 10.1016/j. micpath.2020.104371

Yu, R.-R., Xu, Y., Wang, Y.-G., and Qiu, F.-Y. (2013). Chemical environment influence the incidence of childhood autism. Asian J. Chem. 25, 8835-8837. doi: 10.14233/ajchem.2013.14870

Conflict of Interest: The authors declare that the research was conducted in the absence of any commercial or financial relationships that could be construed as a potential conflict of interest.

Publisher's Note: All claims expressed in this article are solely those of the authors and do not necessarily represent those of their affiliated organizations, or those of the publisher, the editors and the reviewers. Any product that may be evaluated in this article, or claim that may be made by its manufacturer, is not guaranteed or endorsed by the publisher.

Copyright (c) 2021 Zhou, Yu, Ahmed, Jiang, Zhang, Lv, Alhumaydhi, Allemailem and Li. This is an open-access article distributed under the terms of the Creative Commons Attribution License (CC BY). The use, distribution or reproduction in other forums is permitted, provided the original author(s) and the copyright owner(s) are credited and that the original publication in this journal is cited, in accordance with accepted academic practice. No use, distribution or reproduction is permitted which does not comply with these terms. 